\title{
Food \& Nutrition: The driving factors of our gut microbes
}

\author{
Matthias Scholz ${ }^{1}$, Heidi Staudacher ${ }^{2}$, Francesca Fava ${ }^{1}$, Kieran Tuohy ${ }^{1}$ and Kevin Whelan ${ }^{3}$ \\ ${ }^{1}$ Fondazione Edmund Mach, Trento, Italy, \\ ${ }^{2}$ Deakin University, Victoria, Australia and \\ ${ }^{3}$ King's College, London, United Kingdom
}

\section{Abstract}

Introduction: The gut microbiome has attracted much attention in the last decade due to new studies and technologies revealing how gut microbes play a key role in human health and disease risk. However, we still know little about how diets, foods and nutrients shape the gut microbiome.

Materials and Methods: To expand our knowledge about the complex relationship between nutrient intake and intestinal bacteria, we performed a broad dietary study covering diverse foods and nutrient intakes of nearly 100 adults. We analyzed metataxonomic data from 16S rRNA sequencing and correlated the microbial abundances of the top 55 dominant taxonomic groups with collected intakes of 40 food groups and 44 nutritional parameters, as well as degree of adherence to healthy eating patterns. Based on Spearman correlation profiles and hierarchical clustering, we also identified food groups and nutritional parameters that modulate in a similar manner microbial community structure.

Results: We will present preliminary results showing direct effects of specific dietary patterns (e.g. adherence to the Mediterranean style diet), food groups (e.g., fruits, nuts, dairy) and nutrients (e.g. iron, magnesium, zinc) on specific genera of the gut microbiota. We found significant associations between alcohol and the bacterial genus Holdemania. We will show that food groups supposed to belong to the same higher-level food category do not always show the expected effect on the gut microbes, which supports the need for a detailed food classification scheme to investigate dietary effects. Similarly, we found that correlations at dietary and higher food group levels more consistently reflect existing diet:microbe associations from the literature while correlations at the nutrient level are prone to confounding factors only apparent at higher alimentary ontology.

Discussion: Our study aims at a more complete understanding of the complex relations between human diet and gut microbiome community structure. Initial results confirm that both the quantity of a nutrient and the dietary source of a nutrient can both influence associations with gut bacteria. Our results may help to define new strategies for modelling diet:microbe interactions in the gut providing a new valuable tool to assist the nutrition and gut microbiome communities.

\section{Conflict of Interest}

There is no conflict of interest 